\title{
Keyword Index to Volume 11
}

\author{
1 p36 420 \\ $4 q 21.2749$ \\ $6 \mathrm{p} 89$ \\ $7 q 11.22-q 21.12812$ \\ $8 q 21-q 22966$ \\ 9p21-22 121 \\ $14 q 24.1-14 q 24.3$ prelingual 77 \\ $15 q 15497$ \\ $18 q 21.1623$
}

ABCC6/MRP6 gene 215

Activin receptor-like kinase 279

Adams-Oliver syndrome 457

Affected siblings 179

Age grouping 425

Alcohol 687

Aldosterone 395

ALK-1 279

Allele combinations 573

Allele frequency distribution 444

Allele sharing method 17

Alpha satellite DNA 357

Alternative splicing 951

Alu 931

ALX4 457, 892

Aneuploidy 325, 337, 754

Aniridia 163

Anterior segment dysgenesis 163

Anticipation 737

Apo(a) isoforms 693

ApoB 444

Apolipoprotein E 437

Apoptosis 784

Arrhythmogenic cardiomyopathy 69

ARVD1 69

Asperger syndrome 189

Association 395, 819

Association study 23, 659

Asthma 590, 619

Asymptomatic carriers 923

Atopy 590

Attitudes 565

Austronesian 931

Autism 189

Autoimmune thyroid disease 81

Autoimmunity 840

Autosomal dominant nonsyndromic hearing loss 121

Autosomal recessive hypotrichosis 623

Autosomal recessive nonsyndromic hearing impairment 812

Autosomal recessive nonsyndromic hearing loss 77
B-cell lymphomas 416

Balanced translocation 189

Barth syndrome 97

Bi-allelic and microsatellite polymorphisms 705

Bioinformatics 57

Bisulfite 493

BMI 271, 425

Bone dysplasia 639

Bone marrow donor registry 794

BrCa1 210

BRCA1 725

BRCA1 mutation 489

BRCA1/2 464

BRCA2 210, 725, 951

BRCA2 gene 955

C282Y 225

CAG repeat 808

CAG repeat expansion 629

Calcineurin 659

Calsequestrin 888

Cancer 725

Candida 433

Candidate gene 69

Candidate gene prediction 57

Candidate region 189

CARD15 6

Cardiac hypertrophy 659

Cardio-facio-cutaneous syndrome 64, 85 Corpus callosum agenesis 530

Cardiovascular disease 23

Cardiovascular risk factors 845

Cataracts 770

Catecholamines 945

Cationic trypsinogen gene 93

CATSPER2 497

CD11B 884

CDKN2A germline mutations 288

cenM-FISH on synaptonemal complexes 879

Central nervous system 315

Centromeres 357

Cerebellar ataxia 770

CFC 201

CFTR 687

CFTR gene 543

$\mathrm{CGH} 452$

Chaperone 784

Chromosome 11 12, 64, 433

Chromosome 16p11.2 145

Chromosome 1q 585

Chromosome 2 232, 835

Chromosome 22816
Chromosome 7q21-q34 409

Chromosome 9754

Chromosome marker 452

Clavicular hypoplasia 892

Cleft lip and palate 835

Cleft palate 265

Cleidocranial dysplasia 892

clustering 179

CMC 433

CMT1A 170

CNS development 530

$\mathrm{COCH} 744$

coeliac disease 828

COL11A1 185, 265

COL11A2 265

COL2A1 265

Collagen 265

Communication 725

Community genetics 872

Complex disease 573

Complex genetic disorders 179

Complex traits 779

Congenital cataract 375

Conotruncal heart defects 349

Consequences 633

Contiguous gene syndromes 497

Copy number 275

Coronary artery disease 437, 671

Corpus callosum 315

Corticosteroids 619

Cowden syndrome 665

Croatia 535

CROHN'S disease $6,179,237,884$

Cryptic 89

Cryptic chromosome aberration 643

CTLA4 81

Culture 493

Cystathionine $\beta$-synthase (CBS) 23

Cystic fibrosis 385, 687

Cystinosis 503

Data mining 57

DC domain 155

DCC 840

Deafness 744

Deletion 89, 375, 931

Deletion, ring chromosome 749

Desmocollins 623

Desmogleins 623

DFNA23 77

DFNA47 121

DFNA9 77 
DFNB32 185

DFNB35 77

DFNB39 812

DFNB5 77

DHPLC 85, 493

Differential methylation 138

DiGeorge/Velo-cardio-facial syndrome 349

Digyny 972

Dilated cardiomyopathy 97

Diploidy 754

Direct sequencing 858

Discrimination 561

DNA damage 671

DNA polymorphism 444

DNA repair 489

Dominance 710

DYS385 304

E2 931

Early-onset breast cancer 464

Eating disorders 945

Echocardiography 395

Economics 475

EEC syndrome 700

Effective population size 385

Elderly 225

Empirical survey 475

Enlarged vestibular aqueduct 916

Epistasis 573

Epsilon-sarcoglycan 138

Ethical dilemma 872

Ethics 475

Europe 385, 475

European $\mathrm{MH}$ group 342

Exercise intolerance 516

Face recognition 555

Facial pattern 555

Familial breast cancer 633

Familial cancer clinic 633

Familial intracranial aneurysm 737

Familial ligand-defective apoB 100959

Familial Mediterranean fever 50

Family selection 489

$\mathrm{FCH}$ domain 744

FG syndrome 352

First polar body 325

FISH 89, 325, 337, 452, 754, 858

FJHN 145

FKRP gene 923

Flow sorted chromosomes 315

Folate 671

Folate metabolism 245

Forensic genetics 39

Forensic markers 39

Founder effect 288, 916, 931
Founder populations 597

Fourth locus 409

Foveal hypoplasia 163

French Canadian population 585

G4.5 (TAZ) gene mutations 97

Gastritis 380

Gaucher disease 369

Gene conversion 275

Gene-environment interaction 693

Gene-gene interaction 693

Gene-gene interaction 945

Gene localisation 185

Gene localization 816

Gene structure 516

Genealogy 705

Genetic association 840

Genetic collection management 475

Genetic data 561

Genetic diagnosis 342, 959

Genetic diversity 385, 444

Genetic heterogeneity 590

Genetic linkage 112

Genetic polymorphisms 437, 659

Genetic screening 565

Genetic testing 489, 561, 565, 872

Genetics 232

Genome scan 271, 828

Genome-wide scan 112

Genomic deletion 497

Genotyping 357

German population 464

Germline mutations 464

Gliostatin 102

Glycogen storage disease 516

Gout 145

GSMA 828

Guidelines 633

H63D 225

Handedness 779

Haplogroups 535

Haplotype analysis 6

Haplotypes 395, 489, 509, 603, 760, 808, 896

Hardy-Weinberg equilibrium 39

Healthy population 679

Hearing loss 652

Heart 395

Height 271

Hemochromatosis 225

Hereditary cancer 611

Hereditary hemorrhagic telangiectasia 279

Hereditary prostate cancer 17

Heritable 652

Heterozygotes 297
Heterozygous familial

hypercholesterolemia 959

HFE 225

HFE2 585

High frequency 652

Hirschsprung's disease 364

Histological subtype 611

HLA 81

HLA haplotype frequency 794

HMSN 170

HNPCC 725

HNPP 170

Homoallelism 872

Homocysteine 23, 671

Homozygosity 872

Homozygosity mapping 770, 966

Human biobanking 475

Human deafness 816

Human genetics 611

Human population genetics 597

Human spermatoza 337

Huntington's disease 30

Hybrid vaccinia expression system 369

Hyper-lgD and periodic fever syndrome 196

Hypertrophy 395

Hyperuricaemia 145

Hypervariable region 1253

Hypodontia 866

IBD 884

ICAM-1 679

IDDM6 840

Idiopathic pancreatitis 543

IL-4R 884

Immunocytogenetics 879

Imprinting 138, 425, 493

In vitro contracture test 342

Incidence of inborn errors 196

India 253

inflammatory bowel disease 112, 237

Insurers 561

Interaction 573, 693

Interleukin-7 receptor $\alpha 509$

Intermediate alleles 872

Intestinal metaplasia 380

Intra-chromosomal recombination 304

Iron 225

Isolate populations 535

Isolated autosomal recessive optic atrophy 966

Italian population 503

Italy 50

ITGAM 884

Japanese 916

Japanese population 402 
Jewish origin 288

Juvenile hemochromatosis 585

Kidney disease 693

Kidney transplantation 693

Klippel-Feil syndrome 468

Language impairment 89

Laser biopsy 325

Lay people 565

LCA 420

LCA9 420

LDL receptor gene mutations 959

Leber's congenital amaurosis 420

Leigh disease 375

Lens 784

LGMD2I 923

LH $\beta$ gene 402

Life insurance 561

Ligation 357

LINE-1 931

Linkage 81, 271, 409, 420, 425, 433, 590,845

Linkage analysis 17, 237, 585, 779, 835

Linkage disequilibrium 23, 237, 437, 489, 760,819

Linkage disequilibrium mapping 125

Linkage interval mapping 125

Linkage mapping 812

Lipoprotein(a) 693

Lipoprotein phenotype 959

LTC4S 619

Macrodeletion 896

Male breast cancer 210

Male-infertility 497

Malignant hyperthermia 342

Manic depressive illness 597

Marenostrin 50

Marinesco-Sjögren syndrome 770

MCKD 145

Mediterranean 288

Medullary thyroid carcinoma 364

MEFV mutations 50

Meiotic recombination 879

Melanoma families 288

Meniere's disease 744

Mental retardation 352, 770

Meta-analysis 828

Metabolic myopathy 516

Methionine synthase reductase 671

Methylation 493

Methylenetetrahydrofolate reductase (MTHFR) 245, 671

Mevalonate kinase 196

Mevalonic aciduria 196

Micronucleus test 671
Microsatellites 304

Midline defects 315

Midwives 565

Mitochondrial DNA 253, 375, 652, 896

Mitochondrial myopathy 102

MNGIE 102, 547

Molecular pathology 215

MSUD 931

MSX2 457, 892

mtDNA deletions 102

MUC1 380

Mucin 380

Multicolour FISH 643

Multilocus statistics 573

Multiple endocrine neoplasia 364

Multiple mtDNA deletions 547

Multiple sclerosis 509, 573

Multiple testing 573

Multiplex assay 416

Mutation 215, 700, 714, 888, 931, 955

Mutation analysis 185, 457

Mutation origin 503

Mutation spectra 385

Mutational analysis 210

Mutations 196, 369, 402, 468, 503, 543

Myoclonus-dystonia syndrome 138

Myopathy 770

Neocentromere 749

Neonatal hypertrypsinaemia 93

Nephropathy 145

Neurodegeneration 808

Neuropathy 170, 770

Neutropenia 97

Nibrin 297

Nijmegen Breakage Syndrome 297, 416

NOD2 237

Nonhomologous recombination 931

Nonsyndromic hearing impairment 185

Noonan 201

Noonan syndrome 85

Novel mutations 923

NSD1 858

NTD 245

Nuclear inclusions 784

Obstetric cholestasis 232

Oligodontia 866

Oocyte 325

Oogenesis 493

Organic aciduria 97

Osteoarthritis 714

Osteosarcoma 611

Ovarian cancer 210, 955

Overgrowth 665

Ovulatory disorders 402 p16 protein structure-function analysis 288

padlock probes 357

Paired-box gene 468

Paiwan 931

Pakistan 77, 812

Pancreatic secretory trypsin inhibitor gene 93

Pancreatitis 687

Parentage testing 39

Parental age 718

Parietal foramina 892

Partial isodisomy 629

Paternal age 754

PAX1 468

PAX6 mutations 163

PAX9 866

PCR-SSCP 714

PDS (SLC26A4) 916

Pendred syndrome 916

Penetrance 225

PEO 547

Periodic fever 50

Periventricular nodular heterotopia 530

Phosphorylase kinase deficiency 516

Physicians 565

PI3-kinase 665

Plasminogen activator inhibitor 603

PNA 337

POLG 547

Polyamine defect 937

Polyglutamine disorders 872

Polymorphisms 23, 245, 380, 395, 679, $760,840,888$

Pooled analysis 828

Population genetics 39, 444, 760

Population history 304

Postaxial polydactyly 409

Power 819

Predictive DNA-testing 30

Preeclampsia 232

Preimplantation genetic diagnosis 325

Prenatal detection 749

PRINS 337

Professionals 565

Promoter polymorphism 619

Prostate cancer aggressiveness 17

Proteus syndrome 665

PRSS1 687

Pseudogene 516

Pseudoxanthoma elasticum 215

Psychiatric genetics 597

Psychological impact 30

PTPN1 1 201, 201

PTPN11 gene 64

PTT 951 
PXE 215

Pyrin 50

QTL 125

Quantitative assay 170

Quantitative trait 652

Quantitative trait locus (QTL) 845

R3500Q mutation 959

Radiosensitivity 297

Rapp-Hodgkin syndrome 700

Real-time PCR 170

Recessive genetic conditons 652

Recurrent abortion 972

Reduced folate carrier 1 (RFC-1) 245

Reduced penetrance 196

Referral 633

RET mutation 364

Retina 420

Retinitis pigmentosa 155

Risk 725

ROA1 966

Robin sequence 265

Robustness 819

RP1 155

RP1L1 155

RUNX2 892

Ryanodine receptor 342,888

Sardinia 802

SCA17 629

SCKL 851

SCKL3 851

Screening 225

Seckel syndrome 851

Second malignancy 611

Sex ratio 754

Short tandem repeats 304

SHP-2 201

Sib-pairs 590
Single-nucleotide poly-morphism 357, 509, 603, 652

Skewed X-inactivation 352

Slavic 416

Smad3 gene 714

SMN 275

SMN1 275

SMN2 275

SNP 6

Sotos syndrome 858

Spastic paraparesis 710

Spastin 710

Spatial autocorrelation 385

Spatial patterns 385

Spermatozoa 754

Spermine synthase 937

SPG4 710

Spinal muscular atrophy 275

SPINK1 687

SPINK1 gene 543

Spinocerebellar ataxias 808

Spondyloepiphyseal dysplasia tarda 639

Spontaneous chromosome instability 297 Ulcerative colitis 237

Sporadic mutation 718

STAT6 884

Stomach cancer 955

STR database 39

Structural aberrations 754

Subtelomeric 89

Subtelomeric chromosome regions 643

Sudden cardiac death 69

Surname 794, 802

Tandem duplication 497

TATA-binding protein 629

TBX1 349

TCOF1 718

TDT 819

TGF- $\beta 279$
TGF- $\beta$ receptors 279

Thymidine phosphorylase 102

Tissue-type plasminogen activator 603

Tooth development 866

Tooth size 866

TP63 700

Transcription 619

Translocation 530

Transmission disequilibrium test 835

TRAPP 643

Treacher Collins syndrome 718

Tribes 253

Triploidy 972

Trisomy $17 \mathrm{p} 452$

Tristan da Cunha 705

Trypsinogen 687

Tuberous sclerosis 665

Tumour suppressor gene 951

Turkish 851

Twin pairs 845

Type 1 diabetes mellitus 81

Variance component models 125

Variant NBS1 proteins 297

Ventricular arrhythmia 888

Vertebral anomalies 468

Vertigo 744

Weaver syndrome 858

X-linked 639

X-linked mental retardation 937

XLMR carriers 352

Y chromosome 535, 705, 802

$\mathrm{Y}$ chromosome diversity 304

Zn-finger 530 